\title{
Imaging the adult brain
}

\author{
Ivan Moseley
}

The techniques which form the neuroradiologist's armamentarium depend in one form or another on transformation of energy waves; with the exception of ultrasonography, those of the electromagnetic spectrum. None of these energy forms is free from potentially noxious biological effects, and this is particularly true of the socalled ionising radiations, $x$, $\beta$, and $\delta$ waves. It is probably true that, as in the case of new drugs vis-à-vis the formulary, attempts to introduce $x$ ray based techniques as innovative procedures today might well fail. It is therefore realistic to consider diagnostic imaging procedures primarily in relation to the energy source employed: $x$ rays and other ionising radiations, radiofrequency, and sound waves.

\section{Plain radiographs}

The neuroradiologist's use of $x$ ray techniques has changed considerably over the past 20 years. "Radiographic" ( $x$ ray) procedures register the differential absorption of a beam of $x$ rays by the various tissues of the body. They differ greatly in the manner in which that registration is effected so as to preserve anatomical relations and maximise clinically useful data, while attempting to limit radiation exposure. In the classical radiograph or "plain film" the patient is simply placed between a source of $x$ rays and a film sensitive to radiation. Because most of the unabsorbed radiation is emitted in a straight line, anatomical relations are maintained and spatial distortion is minimal unless the film is not orthogonal to the beam; most modern radiographic tables ensure that obliquity is avoided.

\section{Contrast media in plain radiography}

Contrast media can be employed to increase radiographic contrast between anatomical structures, demonstrating some not normally visible. They are usually introduced into preexisting body spaces, such as the alimentary tract or subarachnoid space (for myelography or cisternography) or into the vascular system, where their flow within (in arteriography or phlebography) or excretion from it (in urogra-

Lysholm Department of Radiology, National Hospital for Neurology and Neurosurgery, Queen Square, London WC1N, UK and Department of Radiology, Moorfields Eye Hospital, City Road, London EC1, UK I Moseley tube-patient-film. In $x$ ray computed tomography (CT) the beam emerging from the patient is registered not on a film but by an array of radiation detectors, as the $x$ ray tube passes around the part being examined (in this case, the head). A computer is used to transform the intensity of the emergent beam relative to the incident beam into measures of $x$ ray attenuation, to identify in two dimensions the site of the tissue causing that attenuation, and to construct a two dimensional map of attenuation analogous to an anatomical section. The planes of section of the head given by CT are effectively limited by the construction of the gantry bearing the $x$ ray tube and detectors to axial and coronal planes. Reformatted images in other planes can be constructed by analysing the data from a series of contiguous sections. One of the latest developments in CT is "spiral", or more correctly "helical", scanning, in which the tube rotates continuously around the head while the patient is moved through the gantry. The rapidly acquired, continuous sequence of attenuation measurements can be "cut up" into segments, corresponding to two dimensional sections the thickness of which is determined by the operator. The merits of this method are speed, which makes it more useful for examination of children and uncooperative adults, and greater spatial resolution in images reconstructed in planes other than that of acquisition.

The relative "attenuation coefficients" of certain structures can also be modified by contrast media, usually containing iodine, introduced into the vascular system or subarachnoid space. Other types of contrast medium, such as inhaled stable xenon gas, are rarely used in clinical practice.

\section{Digital radiography}

The third main method of registering $x$ ray attenuation data is by using a high speed camera to scan a screen coated with a phosphor that emits light when radiation falls upon it. If the camera transforms the light energy into an electronic signal, this can be digitised and a computer employed to compare the results of one scan of the phosphor screen with that of those which precede or follow it. A digital to analogue converter can then store and display only the difference between two sets of attenuation data-before and after arrival of contrast medium, for example - in a format similar to that of the original phosphor screen picture, but with unwanted detail removed. This is the
In $x$ ray tomography of all kinds, some motion occurs between the three elements, $x$ ray series of $x$ ray exposures, otherwise similar to those when no contrast medium is used.

\section{Computed tomography}


basis of digital subtraction techniques, used particularly in angiography to produce images in which only the blood vessels are displayed. The major advantages of this technique are that the detection system is much more sensitive than plain film angiography to minor changes in intensity of the emergent beam, and that, because the data are stored on a computer, they are amenable to interrogation and postprocessing.

\section{Radionuclide studies}

The other techniques using ionising radiations generally consist of the introduction into the body of a radionuclide-namely, a source of radiation, usually $\beta$ or $\delta$ rays - and registration of the radiation it emits with external detectors. Originally, these detectors gave no spatial information, but with the development of scanning methods subsequently applied to CT, and the gamma camera, a scintillation detector similar to the phosphor screen device referred to earlier, anatomical localisation became possible, although spatial resolution is inferior to that of most of the techniques described. Modern techniques such as single photon emission computed tomography (SPECT) use a combination of scanning and scintillation detection. Positron emission tomography (PET), to be discussed in a later article in this series, uses the same fundamental principles, but with some technical modifications.

The great potential merit of the radionuclides used in these nuclear medicine studies is that they can be attached to chemical compounds which have a greater or lesser degree of tissue specificity, and provide metabolic or dynamic information.

\section{Nuclear magnetic resonance methods}

These exploit the behaviour of nuclei with unpaired electrons (paramagnetic nuclei) within the body in a strong magnetic field. The basic principle is very simple: some of these nuclei align themselves with that external field and can be displaced by a pulse of radiofrequency energy. The extra energy imparted to the system is dissipated, also as radiofrequency waves, once the radiofrequency pulse ceases and the nuclei return to their previous position. The speed at which the energy is given up, and thereby the strength of the radiofrequency signal at any given moment, depend on the biophysical characteristics of the tissue in which the nuclei (typically hydrogen protons in clinical imaging) lie; the decay of energy release is exponential, the curve being determined mainly by two "relaxation time constants", T1 and T2. For many biological tissues $\mathrm{T} 1$ is of the order of $1000 \mathrm{~ms}$ and T2 $75 \mathrm{~ms}$. The intensity of the energy released from a given tissue depends on the number of susceptible nuclei: water has numerous mobile protons, whereas dense bone has very few. As the frequency of the radio signal emitted also depends on the strength of the magnetic field, a "gradient"- that is, a variation in strength of the field across the region being examined-enables localisation of the nuclei in one plane of space. Thus by examining the intensity and the rate of decay of a radiofrequency signal of known frequency, a number of the characteristics of the proton emitting it can be assessed. Simple though the principle may be, the electronic switching and computation required for three dimensional localisation and tissue characterisation are exceedingly complex.

The characterisation can be refined by modifying the way in which the nuclei are excited, applying a "sequence" of radiofrequency pulses instead of simply one, and by sampling the emitted signal after varying delays. Consideration of how this is achieved is beyond the scope of this article; suffice it to say that most clinical MR images are maps of water distribution, but that they can be modified to show mainly differences in T1 (T1 weighted images) or T2 (T2 weighted images) of the tissues being studied. As a simple guide, the $\mathrm{T} 1$ weighted images give more morphological data, whereas the T2 weighted images provide more information about the constitution of the tissues. A full MRI study usually combines at least these types of image. Some of the unsatisfactory diagnostic applications noted in the early days of MRI resulted from the use of a limited number of sequences, or sequences which did not provide sufficient contrast between normal and pathological tissues. Intravascular contrast media, mainly containing the paramagnetic rare earth gadolinium, can be used, as with $x$ ray $\mathrm{CT}$.

\section{Flow imaging}

Because electromagnetic waves travel at the speed of light, techniques which employ them are essentially instantaneous. Because in MRI there is a finite delay between the application of the excitatory radiofrequency pulse and reception of the emitted magnetic resonance signal, however, protons which are moving through the tissue being imaged have different properties from those which are macroscopically stationary. This enables the relative gross movement in blood vessels and the CSF to be characterised and imaged with specially designed sequences.

Magnetic resonance angiography (MRA) was first presaged in the imaging literature almost a decade ago, ${ }^{1}$ but has been available in clinical practice and of a quality which makes it a useful investigation for about three years; image quality is still improving. A more detailed account of the technique and its applications will be found in a later article in this series. In combination with MRI, clinical application of MRA will bring about a major change in the investigation of at least those patients in whom information about the cervical or intracranial vessels is useful, but not central to their management-for example, patients with parasellar tumours. In the long term, it may completely replace diagnostic angiography. 
The present and future role of CSF imaging is less unambiguous. A number of research papers have been published concerning motion and pulsatility of the intracranial fluid in such puzzling conditions as normal pressure hydrocephalus ${ }^{2}$ and benign intracranial hypertension, ${ }^{3}$ but without as yet casting any clear light on pathophysiology. One reviewer ${ }^{4}$ identified other applications for phase contrast MRI of CSF movement: assessment of the functional significance of drainage via extra-arachnoid structures or the central canal of the spinal cord; investigating why some arachnoid cysts enlarge; and characterisation of obstructive hydrocephalus "without having to introduce contrast agents into the subarachnoid space"-a practice which virtually disappeared with the introduction of CT! In another study, a fast field echo technique, applied to determination of the patency of ventricular shunts, was shown to be fallible, despite which the authors claimed that the technique "may be useful". ${ }^{5}$ The future clinical utility and, indeed, adoption of these applications are at present questionable.

\section{Diffusion weighted MRI}

Magnetic resonance imaging techniques can be used to show other physical characteristics of tissues. One method which has been developed over the past five years is diffusion weighted imaging. ${ }^{6}$ Depending on the gradients used, the contrast in the images can depend on magnetic susceptibility, perfusion, and diffusion. To date the genuine clinical applications of this technique have also not been clarified, although research has been carried out on brain myelination, stroke, and some tumours. ${ }^{7}$

\section{Magnetic resonance spectroscopy}

The phenomena of nuclear magnetic resonance were originally employed for in vitro chemical analysis. In vivo analysis of body tissues can be carried out with magnetic resonance spectroscopy (MRS), which relies on the same variation in the resonant frequency of given nuclei due to their physicochemical environment to create spectra which reflect the concentration of chemical compounds containing them. In the brain, spectra from both hydrogen and phosphorus containing compounds have been investigated; much clinical research on proton spectroscopy is currently oriented towards relative concentrations of choline, creatine, n-acetyl aspartate, and lactate in normal and pathological brain tissues. Magnetic resonance spectroscopy is not strictly an imaging technique, although it employs essentially the same imager; it is combined with MRI to localise the volume of brain from which the spectrum is obtained. Until recently, a major problem with clinical applications of MRS has been the low signal to noise ratio, which has entailed large volumes of interest and long acquisition times. Smaller volume spectra have, however, shown, for example, that choline tends to be increased in solid brain tumours, whereas alanine concentration is high in meningiomas; however, even though such information might be useful in cases in which the imaging features were otherwise inconclusive, spectroscopic results are by no means diagnostic. ${ }^{8}$ Wider application to cerebral metabolic disease is awaited. ${ }^{9}$

Unlike MRA, for which the major future contribution to clinical neurology and neurosurgery seems unequivocal, these other techniques are perhaps still in search of clinical applications.

\section{Sonography}

An ultrasound probe contains a "transducer" which emits 1-20 MHz sound waves and, when they bounce back off the tissues, converts the reflected energy into an electrical signal, which is analysed, usually to form an image. Bone, however, is not a good medium for transmission, so that current applications of sonography to the brain are confined to small children, in whom the fontanelles act as "acoustic windows", patients with skull defects (including intraoperatively ${ }^{10}$ ), and examination of the eye and the major arteries in the neck. The major arteries will be considered in another article in this series.

The use and utility of imaging procedures An article in this journal in May $1994^{11}$ considered the possibility and utility of "periodically updated reviews of all randomised controlled trials relevant to neurology and neurosurgery". Virtually none of the trials referred to was primarily concerned with imaging the brain, and the unfortunate truth is that many clinicians appropriately guided by controlled trials of treatment approach imaging in what seems to their radiological colleagues to be a decidedly aleatoric way, guided by personal prejudices and what their chiefs used to do rather than by the latest audit on best practice. That radiologists are also prone to promote procedures that they enjoy ("cath time" 12 ) and that far too many radiological publications are uncritical in their praise for recently developed procedures ${ }^{13}$ is unhelpful.

My intention is to review, very broadly, categories of disease, and to attempt to indicate which imaging tests are the most likely to provide the information most appropriate for management of the patient; in general, I shall not dwell on how imaging research has clarified disease processes. I shall not look at patients likely to have cerebrovascular disease, as this is the subject of another review in this series. Neither shall I attempt to review all the imaging literature of recent years. Regrettably, in the countries from which the bulk of this literature arises there are strong motives for both promoting the use of medical facilities and for publishing one's work, which may explain the remarkable, and sometimes lamentable absence of outcome studies. To my knowledge, there is, for example, very 
little evidence that the outcome for patients with even the "surgical" neurological diseases (intracranial tumours, abscesses, haematomas, etc) has been appreciably improved by the most recent advances in imaging, except where these are indissolubly wedded to a surgical technique, as in image guided stereotaxic surgery. Indeed, such studies as have been performed have produced very disheartening results, showing no long lasting improvement in the quality of our patients' lives. ${ }^{14}$

Outcome in this sense is, of course, not the only measure. The great advantage of many modern imaging methods is that the patient endures far less unpleasant experiences, or runs less risk, to achieve the outcome, albeit identical. In this sense, CT represented a considerable advance over its predecessors, not necessarily improved on by progression to MRI, with the notable exception of MRA. Even short term outcome may not be affected much. Thus in MRI studies of the brain in patients with AIDS, there is no clear benefit for the large majority if contrast medium is given intravenously, ${ }^{15}$ but some radiologists would defend its routine use. "Some of us are willing to pursue diagnostic accuracy with imaging studies to greater lengths than others, and each of us is likely to have some diseases to which we are willing to devote more time than others. This phenomenon [the "compulsiveness factor"], rooted in human nature, will survive even the current rage for outcome analysis". ${ }^{16}$ One may counter this by observing that radiologists, like too many other doctors, become so accustomed to sticking sharp objects into their patients that they cease to regard it as an assault. Were the doctor injecting a patient to explain that his main motive was diagnostic compulsiveness rather than any expectation of altering management, he might well expose himself to a charge of battery! ${ }^{17}$

With the advent of CT about 20 years ago, followed less than a decade later by MRI, the range of methods by which neuroradiologists make diagnoses and carry out follow up studies has paradoxically decreased. Both these cross sectional imaging techniques have led to a very significant fall in the number of other investigations performed, both plain radiographic and invasive, something which did not occur with the introduction of radionuclide examinations into neuroradiology in the years preceding the introduction of CT. ${ }^{18} \mathrm{We}$ are still going through the phase, familiar to radiologists, in which patients are submitted to multiple tests which yield essentially the same information, as occurred with CT and some of the older invasive investigations. ${ }^{19}$ Thus many patients currently undergo both CT and MRI when either (or even neither) would suffice, but one may hope that this is a temporary phenomenon.

The choices of imaging procedure(s) the clinician has to make when confronted with a given clinical problem were summarised by the Royal College of Radiologists in a booklet Making the best use of a department of clinical radiology. Guidelines for doctors, ${ }^{20}$ the second edition of which appeared at the end of 1993. Its recommendation was that every time someone thinks of requesting an examination they ask themselves "Do I need it?"; "Do I need it now?"; "Has it been done already?"; "Have I explained the problem?"-that is, do the radiographer and radiologist understand what I need to know and why?; and "Is this the best study?". The answers to several of these questions are different in Britain in 1995 from what they might be in an ideal world. Most neurologists and neurosurgeons acknowledge that they are perforce tempered by considerations of cost and availability, as well as inconvenience and risk to the patient (from radiation and other hazards), but they should also be informed by the answers to the implied questions "Is this test really likely to have any impact on my management of this patient? If not, will it nevertheless give data which will genuinely help my understanding of this disease and management of other patients with similar problems?", and, one might add "If so, does the patient who will suffer the inconvenience and risk of this testa threat to life of 1 in 40000 in contrast enhanced CT studies, for example ${ }^{21}-$ know that?"

\section{Head injuries}

The controversy over the optimal imaging approach to head injuries continues to burn and, regrettably, the Royal College of Radiologists' booklet only serves to fan the flames. A major problem with rational management in Britain is that, more than in any other group of potentially neurological patients, the primary care of those with acute head injuries is largely entrusted to inexpert junior doctors who lack both the necessary maturity of judgement and a clear knowledge of the facts. The confusion over the relative roles of plain radiography and other tests, which in this context means essentially CT, pervades the Royal College's recommendations. Complex non-invasive techniques should not be employed to identify fractures of the cranial vault in patients with significant intracranial injuries. The presence of a linear fracture itself is irrelevant to the patient's management. It is often said that lawyers place great emphasis on injuries having been severe enough to cause a fracture; if this is so, it should be the clinician's task to re-educate them. If plain radiographs have any part to play it is in the assessment of complex fractures and, more debatably, ${ }^{22}$ in raising suspicion of an underlying injury which may require treatment. Thus the advice that a patient "disorientated or worse ... drunk/ difficult to assess [or having] stable focal neurological signs" should have a skull radiograph and be admitted for observation seems like recourse to both belt and braces; that consultation with the neurosurgeons be immediate if the skull film shows a fracture but only after 12 hours if the patient fails to improve is questionable at best. Even more debatable is the 
suggestion that for a patient developing focal signs or whose conscious level is deteriorating, even for one whose pupil is dilating, pulse slowing, and blood pressure rising CT or a skull film is appropriate-as though the two were equivalent! A nationwide survey in the United States, reported in 1991, indicated that skull radiographs were obtained for trauma rarely or never in almost half of the hospitals surveyed, and performed less often in larger hospitals, with free access to CT, and at the behest of neurologists or neurosurgeons. ${ }^{23}$

As indicated earlier, CT would at present seem to be the primary investigation for detecting the intracranial complications of head injuries for several reasons: cheapness and general availability, lack of the logistic problems posed by carrying out MRI in emergency situations with a patient on a ventilator, etc, the ready detection of acute haemorrhage, and the fact that the management and prognostic relevance of the changes shown is understood. Magnetic resonance imaging is more sensitive to contusion or shearing injuries, but currently the therapeutic consequences of their demonstration are less well defined. This point applies not only in head injury. Because more intracranial lesions can be shown by MRI than by other techniques in patients with suspected disseminated malignancy, etc, staging of these patients, based on the sensitivities of older techniques, may have to be revised.

A late consequence of head injury which can lead to longstanding problems is the CSF fistula, usually giving rise to rhinorrhoea. A number of investigations, some of which (conventional tomography of the skull base, radionuclide cisternography) deliver a hefty radiation dose for little or no return, have been proposed in the past. For patients who are surgical candidates because of persistent problems, however, CT with intrathecal water soluble contrast medium, performed in the active phase of leakage, ${ }^{24}$ is often the only radiological investigation required.

\section{Brain death}

Innumerable techniques have been proposed for use in the diagnosis or recognition of brain death, often with little or no regard to the relevant national or international recommendations. Indeed, many of the suggestions seem to be predicated on the presumed inability of neurologists to make the diagnosis reliably. The Guidelines for the determination of death drawn up in the United States by the President's Commission almost 15 years ago indicated that the diagnosis is indeed clinical, and that paraclinical tests, including imaging, might be used firstly to determine the cause of irreversible coma and secondly to shorten the period of observation required for definitive diagnosis. ${ }^{25}$ There is little or no evidence that these imaging procedures are in practice used to supplement clinical examination in earlier determination of brain death, their only putative merit. One should not forget that, as
Griner and Glaser ${ }^{26}$ observed, diagnostic tests may be useful in four ways; two of thesescreening and diagnosis-may be of direct utility to the patient. The third-assessment of treatment-is just as likely to be beneficial, if at all, only to the doctor, whereas the fourth-income generation-is uniquely so. The presumably self interested claim in the Royal College of Radiologists' Guidelines that in brain death radionuclide investigations have a "first line" role for "confirmation of neuronal loss" (!) seems as inappropriate as factually incorrect.

\section{Acute lesions of cranial nerves}

The plain film has no part to play in the initial assessment of patients with visual loss, except when this is thought to be due to an intraocular foreign body ${ }^{27}$; neither are there any indications for optic foramen views. ${ }^{28}$ Should examination suggest an ocular cause other than intrinsic disease of the lens or retina, sonography of the globe (a specific technique best reserved for experts) may be employed. Magnetic resonance imaging may produce images which are more readily interpreted by the non-specialist, but it is doubtful that it has much to contribute when expert sonography is available.

When the signs suggest involvement of the intraorbital optic nerve, sonography is contraindicated; CT is probably as useful in most cases as MRI, and may show some significant abnormalities such as calcified, buried drusen of the optic disc or small, or even large, ${ }^{29}$ plaques of calcification in a meningioma of the sheath of the optic nerve, to which MRI is insensitive; MRI may, however, be more informative when CT shows a mass lesion with a questionable anatomical relation to the optic nerve (and therefore its amenability to resection), or when it is important to determine whether a tumour arising from the nerve itself or its sheath extends intracranially.

Lesions within the substance of the anterior optic pathways that are not space occupying, essentially inflammatory, or degenerative, can be shown only by MRI. Thus pronounced swelling and contrast enhancement of the nerve-sheath complex may be shown in sarcoidosis. With high resolution coronal sections, it may be clear that the inflammatory process involves the meninges rather than the nerve itself, and the same contrast enhanced MRI study may show multifocal intracranial meningeal involvement as a bonus. The genuine utility of demonstrating plaques of demyelination, signal change in Leber's optic neuropathy, etc, is debatable, unless the presence and extent of a lesion within the optic nerve could influence the decision whether to treat the patient with high dose steroids. In Britain, about two thirds of patients presenting with classical retrobulbar neuritis will go on to develop multiple sclerosis, and there is evidence that the large majority of those at risk can be identified by the presence of lesions in the brain at the time of initial presentation with visual disturbance ${ }^{30}$; if this 
knowledge is considered important, a stronger argument can be made for requesting MRI of the brain rather than the optic nerves. There are nevertheless some conditions, such as radiation neuropathy, in which imaging may be used not only to exclude a compressive lesion (a recurrent skull base tumour, for example) but also to establish a positive diagnosis, by showing pathological contrast enhancement within the nerve(s). Moreover, as damage to the blood-brain barrier is evanescent, follow up studies showing its restitution can resolve any diagnostic doubts. ${ }^{31}$

Mass lesions confined to the optic canal or affecting solely the adjacent bone are very rare. Magnetic resonance imaging would be the best technique for showing such masses, although CT often shows the bone to better effect. Evidence that surgical or other treatment is effective for visual loss due to trauma to the optic canal region is largely unconvincing, and a recent authoritative review suggests that it should be the subject of a serious controlled trial. ${ }^{32}$ Recommendations about radiological attempts to show fractures, etc, must therefore be viewed critically. It may, however, be important for the faciomaxillary surgeon to be forewarned of the presence of a fracture through the canal when a visually intact patient is to undergo treatment for other facial injuries; this would be best documented by CT. As indicated, MRI is the examination of choice for demonstrating intracranial extension of a primary tumour of the optic pathways, manifest as signal change or expansion of the optic chiasm. With the addition of intravenous contrast medium, it is also the best way of demonstrating spread to the intracranial meninges of an optic nerve sheath meningioma. ${ }^{33}$

If the pattern of field loss localises the visual pathway disturbance to the intracranial optic nerve (by virtue of a contralateral junctional scotoma) or to the optic chiasm, MRI is in general superior to CT not only for detection, but even more so for effective preoperative characterisation. Images can be obtained in multiple planes, and MRA, which adds only a few minutes to the duration of the examination, can adequately show the parasellar arteries. An important study showed that aneurysms of sufficient size to compress the proximal intracranial optic pathways were unlikely not to be visible on MRI or CT. ${ }^{34}$ The investigation of lesions affecting the optic chiasm is essentially similar to that of pituitary tumours, and is considered below. Patients with retrochiasmal visual loss can be divided clinically into two groups: those in whom the visual deficit is isolated and those with other evidence of a cerebral lesion. For a cerebral lesion the role of imaging is to show the presence or absence of a mass lesion, plaques of demyelination, etc, and these are considered elsewhere. An isolated homonymous hemianopia is usually due to an infarct involving the optic radiations or visual cortex, and CT or MRI are adequate for its demonstration. Angiography is not required unless some form of intervention would be contemplated.

\section{Papilloedema}

When patients presenting with longstanding headaches or visual disturbances are found to have bilateral disc swelling, the assumption is that they have papilloedema due to raised intracranial pressure until proved otherwise. From a strictly pragmatic viewpoint, a simple CT study without intravenous contrast medium only is required to establish firstly that there is no intracranial space occupying lesion, and secondly that lumbar puncture, indispensable for the diagnosis of benign intracranial hypertension, may be performed safely. It should be unnecessary to add that the cerebral ventricles are of normal size in this condition. ${ }^{35}$ Demonstration of an empty sella turcica and dilated optic nerve sheaths is a bonus. MRI will show the sheaths in more detail, which may conceivably be useful if visual impairment is such that operative decompression of the sheaths is considered. It can also give additional information on the patency or otherwise of the dural venous sinuses, but the true value of that information in someone with chronic disease is highly questionable; the employment of invasive angiography can scarcely be justified.

\section{Acute ocular motor nerve palsies}

The investigation of acute cranial nerve palsies presumed to be due to structural vascular lesions, specifically aneurysms, will be discussed in a later article in this series. Many of the larger non-vascular lesions, particularly those amenable to treatment, will be visible on CT but, in as much as isolated acute cranial nerve lesions may be a manifestation of demyelination, MRI is clearly preferable. ${ }^{36}$ Even then, no causative lesion will be shown in most patients presenting in this way; modern imaging has largely failed so far to elucidate such lesions as the "microvascular" third nerve palsy.

Extending the CT or MRI study to the orbit can be of value in identifying lesions of the superior oblique or lateral rectus muscles that mimic fourth or sixth nerve palsies.

\section{Acute facial palsy}

There have been reports of positive findings on MRI, particularly with gadolinium contrast enhancement, in patients with isolated acute facial weakness, and their relation to prognosis. ${ }^{37}$ In typical cases of Bell's palsy, however, the genuine therapeutic utility of these expensive investigations may be on a par with that of showing uncomplicated rib fractures with a chest radiograph, and it seems entirely reasonable to reserve imaging for atypical cases. One must be aware that, as with some inflammatory lesions-cerebral abscess being a prime example-contrast enhancement may persist once clinical resolution has occurred. One must remember that 
imaging can reveal only a relatively small range of abnormalities (swelling, shrinkage, change in density or intensity, etc); it cannot interpret images without the clinical picture firmly in mind, as a report of findings on contrast enhanced MRI in the Ramsay Hunt syndrome- in a patient with symptoms for two days-mimicking an intracanalicular vestibular schwannoma ${ }^{38}$ emphasises.

Acute lower cranial nerve palsies are commonly either vascular or demyelinating and are therefore treated elsewhere.

\section{Chronic lesions of the lower nerves}

The number of patients presenting with disturbances of hearing or balance is very large, and were all to be intensively investigated, the imaging services would be in danger of being swamped. The number of patients referred for imaging can, however, be controlled so that the services are used much more appropriately if neuro-otological consultations precede imaging. This is particularly true in patients who have vertigo without hearing loss, in whom a positive diagnosis can often be made without recourse to imaging, and a structural lesion is rarely found. When the history, examination, and otological tests indicate the possibility of a vestibular schwannoma, there is now little justification for carrying out any test other than MRI. Plain films and conventional tomography have no part to play in the 1990s (and it was very difficult to rationalise their use in the preceding decade). ${ }^{39}$ It is now evident that, when MRI is not available, CT is a poor second best, although it can demonstrate relatively large tumours. The promotion of gadolinium enhanced images for MRI demonstration of smaller tumours ${ }^{40}$ was always questionable, as most could be shown with adequate clarity by a combination of $T 1$ and T2 weighted images, except perhaps on the least satisfactory machines. The recommendations for the use of contrast medium by the 1991 NIH Consensus Development Panel, not published until $1994,{ }^{41}$ have been overtaken by technical developments: it is now widely accepted that high resolution T2 weighted images of the region of the internal auditory meatus in a single plane are adequate for exclusion of even small tumours ${ }^{42}$; when the results are equivocal, addition of a second plane is quicker, kinder, and much cheaper than subjecting the patient to an intravenous injection. Optimal techniques may, of course, vary depending on local factors such as machine strength, availability of specific coils, high resolution programmes, etc; discussion with the radiologist may be necessary to achieve the optimal results in any given unit.

"Vascular compression syndromes" in the posterior fossa continue to stir up debate. If one believes that compression of the fifth or seventh cranial nerves by relatively small vessels can produce facial pain or twitching respectively, and that surgery is the answer, high resolution MRI similar to that used for investigation of the eighth nerve is the optimal imaging technique. It is the only one which shows clearly the cranial nerves and the adjacent vessels: it may be supplemented by MRA showing the whole course of the "offending" artery, ${ }^{43}$ but if the surgical approach does not depend on the detailed vascular anatomy, this may be redundant.

The diagnostic yield of CT in patients with palsies of the lower cranial nerves (IX-XII) is sufficiently low compared with that of MRI that the older technique should be foregone whenever feasible. Intrinsic lesions-neoplastic, vascular, or inflammatory-of the lower brain stem, often quite small, can often be identified only with MRI. ${ }^{4}$ Most extra-axial lesions are also clearly visible, with the exception of malignant or inflammatory basal meningitis, in which even quite extensive lesions may be evident only on images after intravenous gadolinium.

When extra-axial masses are identified in the lower part of the posterior cranial fossa, MRI will confirm the nature of aneurysms or provide preoperative vascular mapping in the case of soft tissue lesions. When MRI reveals a lesion in the region of the foramen magnum, however, particularly one showing inhomogeneous contrast enhancement that suggests the presence of large vessels within it, an intraarterial study with the aim of proceeding to therapeutic preoperative embolisation is strongly indicated.

Magnetic resonance imaging is also the prime investigation for patients in whom brain stem or cerebellar disorders are thought to be due to anomalies at the foramen magnum. Numerous studies have suggested that the cerebellar tonsils may, in normal subjects, extend a few millimetres below the foramen magnum, whereas older studies, usually myelographic, suggested that this was not the case. More recent work suggests that the older data, although obtained with a technique liable to alter the craniospinal hydrodynamics, were indeed correct, and that it is the normal biventral lobules extending through the central part of the foramen, which lie higher than its lateral borders, which give the impression of cerebellar ectopia. ${ }^{45}$ In practice this means that it is not abnormal for a midline sagittal image to show the inferior part of the cerebellum extending slightly below the plane joining the anterior and posterior lips of the foramen magnum. More important pathological observations are that the neuraxis seems to pack the foramen and that the anterior surface of the medulla seems compressed against the odontoid peg. Needless to say, the appearances of "cerebellar ectopia" in a patient without appropriate symptoms or signs are usually irrelevant. Imaging for suspected foramen magnum lesions should include the upper cervical spine, as an asymptomatic associated syrinx may be detected. Plain films or CT may be required before surgery for foramen magnum abnormalities, because of their superior demonstration of the bone.

Acute cerebral and cerebellar lesions It is only a few years since editorials published 
in a number of neuroscience journals emphasised that, despite the inroads of MRI, the diagnosis of multiple sclerosis was essentially clinical, backed up by neurophysiological and CSF testing. ${ }^{46}$ The same can be said of many diseases, especially those in which initial clinical presentation is protean and subsequent course equally variable. In pragmatic terms, however, one may question this judgement. In the appropriate clinical context, particularly in young people, the demonstration of multiple, characteristic lesions, usually in the brain, but occasionally solely in the spinal cord, gives a sufficiently strong clue to the diagnosis to obviate further investigation. For example, the predictive value of a positive MRI study of the brain in patients presenting with retrobulbar neuritis or myelitis as an initial neurological disturbance is so strong that extensive additional investigations would seem meddlesome unless there are discordant features. Whether the patient with optic neuritis should undergo MRI of the brain is an ethical question; in purely pragmatic terms, the efforts of the popular press in drawing attention to this condition as the harbinger of multiple sclerosis are such that many young women consider themselves inadequately investigated without MRI.

It is certainly no longer effectively the case that multiple sclerosis is a diagnosis of exclusion. For both clinical and technical reasons, however, routine MRI is more likely to show lesions in the head than in the spinal cord; indeed, the detection of lesions in the spinal cord is one of the most stringent tests of the quality of the imager. Estimates of the proportion of patients in whom MRI will show spinal lesions when images of the brain appear normal vary, but in the most reliable series the percentage is small. This being the case, a strong argument can be made for imaging the head first and foregoing images of the spine in young patients with relapsing symptoms, when cranial MRI clearly shows typical, disseminated disease. Even when a spinal cord episode is the first neurological illness, demonstration of brain lesions increases the relative risk of progression to multiple sclerosis in less than two years by 36 times, or by about 15 times if patients with complete transverse myelitis are excluded. ${ }^{47}$ The proportion of this group, when attacks involve different spinal levels, in which an additional structural lesion will be found is minuscule. As an analogy, when a patient with multiple enlarged lymph nodes is shown by biopsy to have classic sarcoidosis, one does not proceed to sample all the other nodes in case one might show Hodgkin's disease. In older patients with progressive symptoms, which might be due to superimposed spondylosis, for example, the situation is obviously different.

It is axiomatic that on MRI the lesions of multiple sclerosis are not pathognomonic. Some of the diseases that may simulate multiple sclerosis, including sarcoidosis and tropical spastic paraparesis, ${ }^{48}$ may produce very similar radiological appearances. Clinical features, together with CSF analysis, are therefore paramount in a proportion of cases.
Fortunately, these conditions are rare, as are the often suggested but rarely documented cerebral vasculitides, although these tend to produce more peripheral and basal ganglion lesions, ${ }^{49}$ as does Behçet's disease, which also shows a predilection for the brain stem. ${ }^{50}$ Small vessel ischaemia, however, is ubiquitous, and in the individual patient, where statistical analyses are unhelpful, it may be difficult to make a firm diagnosis on imaging grounds. Lesions which are predominantly periventricular, involving the corpus callosum, and with transverse diameter greater than their anteroposterior extent, favour multiple sclerosis, as does finding multiple, apparently asymptomatic supratentorial and infratentorial abnormalities in a young patient. None of these characteristics is absolute; I am not convinced that statistical analysis of lesion numbers, etc ${ }^{51}$ helps any more than an experienced radiologist's impression in any given case. Once the diagnosis of multiple sclerosis is established or strongly suggested, repeated MRI studies to demonstrate the appearance of new lesions are rarely indicated, particularly in establishing a differential diagnosis between, for example, small vessel ischaemic disease and multiple sclerosis in a middle aged person. Occasionally, the finding of new lesions may facilitate the differentiation of acute disseminated encephalomyelitis, a monophasic illness, and multiple sclerosis; whether this is worthwhile clinically is, however, debatable. In established multiple sclerosis, there is little point in repeat MRI studies charting the course of the individual patient's disease, unless there are strong reasons for thinking that a new neurological episode is due to some other condition.

Before the advent of MRI, some workers proposed double dose, delayed contrast enhanced CT for detection of cerebral plaques, ${ }^{52}$ but this was always of doubtful value as most of the patients with positive results had diagnostic changes in the CSF. Accepting the caveat that the diagnosis of multiple sclerosis is essentially clinical, and that the role of radiology before the advent of MRI was largely exclusory, the use of this technique in patients who cannot for some reason undergo MRI is highly debatable. In general it is also true that when MRI studies are positive in patients with suspected multiple sclerosis, recourse to gadolinium enhancement is unnecessary. Occasionally, however, when alternative diagnoses seem likely, the absence of contrast enhancement of any lesion may strengthen the suspicion of multiple sclerosis, particularly when the patient is not in an acute relapse, whereas enhancement of all or most of the lesions visible on T2 weighted images may point to acute disseminated encephalomyelitis - in which some of the lesions are often large, ${ }^{53}$ - or an inflammatory condition such as sarcoidosis.

Intracranial infections

There is little doubt that CT revolutionised 
the neurologists' and surgeons' approach to many intracranial infections, including cerebral abscesses, empyemas, granulomatous disease, etc. It is also clear that for certain types of infection, notably encephalitis, MRI is significantly more sensitive, particularly in the early stages of the disease. ${ }^{54}$ This is also true of other conditions, such as meningitis and AIDS, but the added sensitivity in some of these may simply allow observation of disease progression rather than conferring any benefit on the individual patient, much though they may advance medical knowledge.

Many subacute or chronic infections produce changes that can be identified on $\mathrm{CT}$, or with greater ease on MRI. Unfortunately, as in so many situations, the radiological findings are non-specific; thus the diagnosis of intracranial Whipple's disease, Lyme disease, ${ }^{55}$ etc, is invariably based as much on clinical and epidemiological factors as on imaging. This also applies to opportunistic infections in AIDS: although in many parts of the world the major organism is toxoplasma, cryptococcus may be relatively common in some areas, ${ }^{56}$ whereas in Spain, for example, tuberculosis is an important consideration. ${ }^{57}$ Differentiation of toxoplasmosis from lymphoma by means of MRI is sufficiently unreliable for antimicrobial treatment or biopsy to be the recommended course ${ }^{58}$ rather than reliance on the radiologist's diagnostic "compulsiveness".

\section{Chronic and progressive syndromes} CEREBRAL LESIONS

Historically, one of the fields to which most effort has been directed in imaging the head is that of progressive focal or generalised neurological deficit-that is, detection and characterisation of intracranial tumours. This is not, however, a single task, and for practical purposes, it can be subdivided as follows ${ }^{59}$ :

- detecting a mass lesion within the head

- indicating whether it lies within or without the brain

- whether it requires biopsy for tissue characterisation

- indicating the best means of intervention

- directing that intervention

- monitoring the natural history of untreated lesions

- secondary effects of the lesion

- the effects of treatment.

It is a truism that skull radiographs and radionuclide investigations are not indicated, even as non-invasive screening tests, for detection of intracranial masses; both have unacceptably high false negative rates. ${ }^{6061}$ Magnetic resonance imaging yields a significantly higher detection rate than CT, especially in the anatomical sites to which CT is known to be relatively blind-namely, the anteroinferior part of the middle cranial fossa and the posterior cranial fossa, particularly the brain stem. Studies carried out when MRI was first introduced, however, gave conflicting results as regards the detectability of supratentorial neoplasms other than in the tempo- ral lobes. ${ }^{62}$ Some of these were methodologically questionable, and more recent scientifically conducted comparisons are few, but a clinical impression is that, with modern equipment, the detection rate for tumours in or adjacent to the cerebral hemispheres with the two techniques is not greatly different. Even clinicians' awareness of this does not, however, prevent many patients having both tests.

Contrary to a widespread belief, numerous studies have confirmed the radiologists' impression that there is only a very small (less than $1 \%$ ) decrease in detection rate of primary cerebral tumours if intravenous contrast medium is not given; furthermore, this was true even when image quality was much inferior to that available today. ${ }^{63}$ It is recognised that "false negative" CT studies occur with fast growing malignant gliomas, particularly in patients presenting with seizures; a second examination months later, by which time neurological signs have often developed, may show a large tumour genuinely invisible on the first ${ }^{64}$; the same may also occur with MRI. Not surprisingly, contrast enhanced MRI has been found to be more effective than CT for detection of intracranial metastases. This applies particularly to extra-axial deposits, to which CT is notoriously insensitive, ${ }^{65}$ but is also true when only parenchymal lesions are present ${ }^{66}$; there seems to be little or no benefit in delaying the postinjection imaging, a manoeuvre claimed to increase the detection rate in CT.

Once the radiologist has decided that an intracranial mass is neoplastic, which is not difficult in most cases, his long term aim would clearly be to be able to provide a tissue diagnosis sufficiently reliable to obviate biopsy. Until we reach that happy state, it can be argued that the neuroradiologist should rather "ask himself whether or not a precise diagnosis is really useful", ${ }^{67}$ and throw in the towel. Situations which engender negative answers to that question are almost certainly more common than otherwise. Exceptions include the fundamentally important, but often rather low level decision, as to whether a mass lies within or outside the brain, as in the former case it is potentially resectable; whether multiple or less commonly single masses are likely to be part of disseminated metastatic disease, in which case surgery on the head, even biopsy, may not be the preferred option; or whether it might be a lymphoma, in which case one should abstain from steroid treatment before biopsy. ${ }^{68}$ It seemed at one time that immunolocalisation of brain tumours, using tissue specific antibodies labelled with radionuclides ${ }^{69}$ or some compound detectable by magnetic resonance, would obviate radiological diagnostic guesswork, but that promise does not seem as yet to have been fulfilled.

Similar remarks apply to attempts to grade degrees of malignancy by imaging. Considerable effort has been devoted to establishing correlations between malignancy and radiological criteria derived from angiography, 
CT, MRI, and isotope studies. When these involve complex formulae or multifactorial analyses which barely achieve statistical significance, ${ }^{70}$ their clinical utility is effectively nil. Simple Gestalt analyses of some of these tests, however, can contribute to prognosis when biopsy is either unhelpful or patently at variance with the radiological data, indicating that atypical tissue has been obtained. These should be the minority of cases, as histological examination is in general more reliable. Unfortunately, many recent enthusiastic reports on radiological differential diagnosis of intracerebral or intraventricular ${ }^{71}$ tumours have the same fatal flaw; the differential diagnostic game may be entertaining for the radiologist to play, but clinical features may be equally important and the images rarely supplant biopsy. It is perhaps puzzling that a report showing a close correlation between grades of malignity of intracranial tumours and the methyl and methylene peaks of peripheral blood on $\mathrm{MRS}^{72}$ seems to have engendered little excitement among neurosurgeons-or perhaps not!

Where extracerebral tumours are concerned, the value of current imaging techniques in general is in similarly providing morphological detail rather than refining diagnosis. Magnetic resonance signal characteristics, for example, do not seem particularly useful for assessing preoperatively the likely consistency of meningiomas. ${ }^{73}$

Surgical use of imaging technology for localisation of lesions to be removed or biopsied has progressed in less than 20 years from the complex but rather crude, using very rough data given by air studies and angiography, to the highly sophisticated, using a variety of techniques and combining the information into computer generated images which often contain more information than the surgeon can use. The ways in which CT or MRI guided stereotaxy is currently performed are almost as various as the institutions involved. Suffice it to say that by the time these words are published routine intracranial surgery carried out, perhaps by remote control, within a dedicated MR imager, with effectively real time data presentation, may no longer be science fiction.

Angiography remains part of the preoperative investigation of patients with intracranial tumours in some institutions, although the benefits it confers are debatable. There would seem to be no justification for using an invasive technique with a known morbidity for localisation of masses, particularly when, as in the large majority of cases, non-invasive procedures are more reliable. Grading of intrinsic tumours on the basis of pathological vessels, etc, has not been shown to be more sensitive than grading by CT or MRI. When information about patency of dural sinuses, etc, is thought necessary before surgery, this can be acquired by intravenous digital subtraction angiography or MRA, and the same may be said for the demonstration of large vessels before stereotaxic biopsy. Virtually the only firm indication for preoperative intra-arterial angiography is when intravascular intervention is foreseen. Presurgical embolisation of some vascular neoplasms, especially those around the skull base such as glomus jugulare tumours or juvenile angiofibromas, is today generally considered the best practice, but surgeons' universal enthusiasm for intra-arterial treatment of, for example, convexity meningiomas, seems to have waned as doubt has been cast on the real benefits. ${ }^{74}$ Unfortunately, meningiomas arising from the skull base, in which surgical access to the feeding vessels is less straightforward and haemostasis would be a real gain, are often not strikingly vascular on angiography. ${ }^{75}$

If cerebral tumours are treated by biopsy and radiotherapy (with or without other types of treatment) rather than by excision, it is important to know the extent of brain involvement, as it is undesirable to expose normal brain to therapeutic doses of radiation. There is evidence from surgical biopsies, postmortem examinations, and correlation with PET studies of cerebral metabolism that CT underestimates tumour spread, ${ }^{76}$ and the same is almost certainly true of MRI. There have been a number of accounts of treatment of cerebral ${ }^{77}$ and brain stem neoplasms by intra-arterial infusion of cytotoxic agents. Theoretically at least, delivering the noxious agent close to the vascular bed of the tumour has advantages, but it remains to be proved that these outweigh the morbidity of the technique.

Computed tomography or MRI are excellent methods of following up patients with cerebral tumours, treated or untreated. It is, however, reasonable to restrict the use of these or any other investigations to clinical situations in which there is some realistic likelihood that they will modify management. When surgery seems inappropriate for a patient with a low grade intrinsic tumour, imaging follow up is required only when a significant change in clinical status occurs, which might tip the balance in favour of surgery, or in the unlikely event that some new operative technique renders intervention a better choice. Much the same applies in many situations; imaging as occupational therapy for the outpatient whose recent clinical status is unchanged some months or years after total removal of a meningioma, for example, is fruitless (Hodgson, Kingsley, and Moseley, unpublished data).

Differentiating recurrent tumour from radionecrosis remains difficult with imaging; neither CT nor MRI is sufficiently reliable. There have been several reports of the use of PET for this purpose, but its expense and limited availability tend to exclude it from routine use; SPECT techniques, which are considerably cheaper, may be informative, however, in at least some cases. ${ }^{78}$

\section{HEADACHE}

A number of surveys have confirmed the very low diagnostic yield of imaging in patients with either non-specific headache unaccompanied by neurological disturbances 
or recognisable forms of migraine, migrainous neuralgia, etc. This is one area in which sensible clinical assessment is invaluable, given the frequency of complaints of headache and the desire of many patients to be investigated. The other side of the coin is that CT is now a cheap and almost completely anodyne test, provided that contrast medium is avoided. When CT was considerably less widely available and relatively more expensive than today, one audit of its utility suggested that an EEG might be a good pre-CT screening test in patients with chronic headache ${ }^{79}$; one could probably reverse this argument on cost-benefit grounds today. Plain films of the skull are an even cheaper way of confirming the diagnosis in unusual conditions in which the skull itself is the source of pain, such as Paget's disease. In a more common situation, films of the paranasal sinuses will usually show or exclude inflammatory or other changes severe enough to cause chronic headache, although they may not suffice for further management. Conversely, plain films of the craniocervical junction or cervical spine hardly ever elucidate isolated occipital pain; moreover, they are so often abnormal in elderly people ${ }^{80}$ that they cannot genuinely support a diagnosis of cervicogenic pain.

Investigation of types of headache known to be associated with intracranial or craniocervical lesions - as in subarachnoid haemorrhage or cough headache-will depend on the presumed nature of the underlying lesion: an intracranial aneurysm or cerebellar tonsillar ectopia, for example. Because, save for such cases, there is little to indicate that MRI is superior to CT in the investigation of headache, it is probably unjustifiable to use the more expensive method as a primary imaging test.

\section{EPILEPSY}

The contribution of imaging to investigation of patients whose illness is dominated by or consists primarily of epileptic attacks has traditionally been the detection of potentially treatable causes, typified by intracranial tumours or vascular malformations. With the advent of CT, causative lesions not amenable to such treatment-small cortical infarcts, or the effects of previous trauma-could be identified. This may have satisfied the neurologist's curiosity, but positive effects on management were minor. ${ }^{81}$ When the increased sensitivity of MRI to parenchymal pathology first became evident, it was suggested that the detection rate for lesions responsible for epilepsy was very much higher than when using CT. This has not been entirely borne out by subsequent experience and, with some exceptions, the abnormalities to which the newer technique is indeed more sensitive tend to be those in which their presence explains why the patient should have fits rather than those to which present surgical approaches are suited.

Thus when a patient presents with seizures, the aim of imaging should be firstly to determine whether he or she has a lesion that should be treated itself, and of which the fits are simply the clinical manifestation. A convexity meningioma is the paradigm; in a relatively young patient the likely morbidity of investigation and treatment is outweighed not merely by the presentation, but also by the natural history. When preliminary imaging shows a complex arteriovenous malformation in an eloquent area in someone of middle age, the equation is more finely balanced, but will depend to some extent on imaging data. Needless to say, plain films and radionuclide investigations are sufficiently insensitive as to be effectively useless, but there is little evidence that for detection of such "surgical" lesions MRI is superior to CT, although further investigations may be required before surgical decision making. When CT is normal in patients whose epilepsy is not so severe as to require more radical surgery, there is little or no justification for further investigation. There are some adults and children in whom MRI will show anomalies of cerebral cortication, or heterotopic grey matter, etc, but if, given today's surgical possibilities, a mildly affected patient would not be a candidate for cerebral resection surgery, this type of investigation cannot be justified in clinical practice, other than as research.

When the fits are so intractable, however, or the side effects of conservative treatment so onerous that the patient would be offered surgery as primary treatment, MRI is the examination of choice. This is particularly true when the presumed epileptogenic focus lies in the temporal lobe, to which CT is recognised to be relatively blind. Thin coronal $\mathrm{T} 1$ and $\mathrm{T} 2$ weighted images, which permit volumetric analysis and three dimensional reconstructions, can show not only hamartomas and indolent tumours, but minor degrees of hippocampal atrophy and signal change, indicating mesial temporal sclerosis. As always when images are used to generate measurements there are important methodological sources of error, from which a number of the most often cited publications may not be free. ${ }^{82}$ Even when these are taken into consideration, for successful surgery the results must be considered in combination with clinical and electrophysiological data, including preoperative tests of cerebral dominance using intra-arterial sodium amytal. Possibly because of the expense of both techniques, the combination of MRI with magnetoencephalography, ${ }^{83}$ to produce what at least one radiologist, possibly with political foresight, has termed "magnetic source imaging", has not become widespread as yet. It has been suggested that interictal or particularly ictal and periictal functional radionuclide studies give more demonstrative lateralising data ${ }^{84}$ but, even so, MRI demonstration of detailed anatomy greatly assists the neurosurgeon. A few years ago, some centres were reporting successful outcomes of surgery for mesial temporal sclerosis in as few as $60 \%$ of patients. ${ }^{82}$ This should improve with current MRI techniques, and in the near future intraoperative MRI will almost certainly prove 
extremely valuable in guiding the extent of resection.

\section{MOVEMENT DISORDERS}

Regrettably, static imaging methods, concerned with structure rather than function, have contributed little to clinical investigation of involuntary movements, except where these are symptomatic of structural disease, as in the rare meningioma causing tremor. Demonstration by high field MRI of putative changes in brain iron distribution in parkinsonism variants was very enthusiastically promoted some years ago, ${ }^{85}$ but has since spectacularly failed to change clinical practice.

Some disorders, such as HallervordenSpatz disease, have highly suggestive MRI findings (the "eye of the tiger" globus pallidus). ${ }^{86}$ The findings in Wilson's disease, however, are variable, inconstant (although much less so than with CT), and do not necessarily correlate with clinical status or response to treatment ${ }^{87}$; they may serve to strengthen a clinical diagnosis, but clearly do not replace metabolic investigations. The atrophic changes seen in "multiple system atrophy" are similarly non-specific and bolster clinical impressions rather than throwing up unsuspected diagnoses. ${ }^{88}$

\section{DEMENTIA}

Investigation of patients presenting with or found to have dementia is highly variable in practice, although the underlying controversy is rarely articulated. Published evidence is certainly contradictory. On the one hand, there are studies, often initiated by radiologists, which show the very poor cost-benefit gains from imaging in such patients, especially those without neurological signs and that, contrary to widespread belief, the proportion of patients with genuinely treatable lesions does not increase greatly with earlier onset of dementia. ${ }^{89}$ On the other hand, hardly an issue of a self respecting neurological journal appears without an article on changes that may be shown by detailed analysis of expensive investigations (CT, MRI, SPECT, or PET) in demented patients. Few, if any, of these have shown any useful, reliable correlation between structural or even functional imaging and specific causes of dementia (rather than dementia in general); indeed, in most published studies the accepted diagnosis is that made on clinical and psychometric grounds. This is especially regrettable, as, despite some claims to greater accuracy, at least one series, in which clinical, radiological, and neurophysiological assessments were compared with postmortem findings, revealed only $47 \%$ sensitivity and $65 \%$ specificity in diagnosing Alzheimer's disease. ${ }^{90}$ It may be true that other neuropsychologists are better at clinical differential diagnosis, but those who have not put themselves to the test should not assume this to be the case. Nevertheless, the frequency and "scientific" tone of such studies seem to have convinced some neurologists that they should be carrying out these tests as diagnostic aids on their patients. Were some specific treatment for Alzheimer's disease, for example, to become available, it would be comprehensible that an imaging test of high specificity and sensitivity might be used to select which demented patients were treated. But unless that test were quick, cheap, freely available, and virtually infallible, it would seem unethical not to extend treatment to other demented patients, unless it proved unacceptably toxic or expensive; and neither that test nor the treatment are currently to hand. It has been suggested that simple linear measurement of the distance between the medial border of the uncus on the two sides might be useful for identifying patients with Alzheimer's disease, but some groups have been unable to distinguish patients with mild to moderate dementia from normal subjects of the same age with this criterion. ${ }^{91} \mathrm{~A}$ recent update on the OPTIMA group's suggestion that measurement of the minimum width of medial temporal lobe tissue at the level of the brain stem ${ }^{92}$ indicates that this simple test does have high discriminant value; however, it is by definition not useful until the disease has already reached a stage at which structural changes have occurred.

It cannot be overemphasised that loss of brain volume is a normal phenomenon in elderly people, and its demonstration cannot be assumed to have advanced the investigation of dementia. For this reason many radiologists prefer the term "cerebral involution", because of the traditionally "pathological" overtones of "atrophy". It should also be understood that correlations between the lesions due to small vessel ischaemia which can be shown by CT or, with greater sensitivity by MRI, and cognitive decline is sufficiently vague to be of no clinical value in the individual case, particularly in the elderly, mildly demented patient. ${ }^{93}$ In a number of brain diseases known to be associated in some cases with dementia (such as parkinsonism), although some workers have suggested focal atrophy of the medial frontal lobes and around the third ventricle, ${ }^{94}$ others have found no good correlation with specific imaging changes. ${ }^{95}$ Not surprisingly, some correlation between cognitive decline and the extent of brain disease has been identified in multiple sclerosis. ${ }^{96}$

It was to be hoped that modern imaging might prove more helpful than invasive techniques in diagnosis of early Huntington's disease, but clinical features and, of course, family history and DNA analysis seem to be better guides. It has been suggested, however, that the degree of atrophy of the caudate nucleus may be a guide to prognosis. ${ }^{97}$

A persistent controversy concerns the frequency (perhaps even the existence) of normal pressure hydrocephalus. In Britain this is a rather infrequent diagnosis in patients without a clear antecedent history of intracranial bleeding or infection, and very small numbers are subjected to shunt procedures on the basis of a diagnosis of idiopathic normal pressure hydrocephalus, but one report describes 22 patients in whom normal pressure 
hydrocephalus had been diagnosed undergoing a non-standard MRI procedure before operation in a relatively short time at a single German university hospital. ${ }^{98}$ When the clinical and laboratory criteria for the diagnosis seem so geographically variable, assessment of claims about the therapeutic significance of putative radiological signs is virtually impossible. With recent reports about the relevance of MRI findings of concomitant white matter disease and arterial hypertension to the pathophysiology, ${ }^{99}$ water on the brain is probably at its muddiest ever, at least in the imaging literature. ${ }^{100}$

\section{OTHER NEUROPSYCHIATRIC DISORDERS}

Several imaging studies in recent years have applied CT, MRI, and radionuclide methods to patients with schizophrenia, autism, psychosis, etc. ${ }^{101}$ The range of imaging features examined has been extensive and various, and few groups have used widely accepted criteria. In addition, many workers have published the statistical analyses of their findings rather than the measurements themselves, which are essential for replication and comparison. The main thrust seems to have been to show that people with psychiatric disorders do indeed have structural abnormalities, a fact which some psychiatrists, presumably battling with their psychoanalytical colleagues, find "exciting". ${ }^{102}$ As yet, the data are too soft and contradictory for any clue to pathophysiology to have emerged, and their clinical relevance is doubtful.

\section{Neuroendocrine disease}

The history of the application of imaging to the investigation of neuroendocrine disease, specifically to small but hormonally active pituitary tumours, makes entertaining reading. As each of a series of techniques has become available, it has made clear the high level of self deception by both radiologists and endocrinologists necessary for the use of its predecessor. Thus plain radiography, conventional tomography, and even $\mathrm{CT}^{103}$ have all been shown to be extremely unreliable in the light of later technology; the particularly high inaccuracy of CT was recognised even before comparisons were made with MRI. ${ }^{104}$ Although MRI is also not infallible, it would seem to be by far the best investigation currently available. As I have pointed out previously, however, "the desirability of locating the tumour within the pituitary gland of a patient with hyperprolactinaemia is predicated solely and absolutely on surgery being the treatment of choice"; it is otiose when chemotherapy will be employed.

When larger pituitary region tumours are present, the multiplanar capacity of MRI, linked to MRA for demonstration of the vascular anatomy, renders this the technique of choice. Combining image quality with economy, in ideal circumstances, it is probably necessary to use only a fat suppressed contrast enhanced $T 1$ weighted volume acquisition but once again this will depend to some extent on the technical possibilities of the apparatus available. This simple technique can also be used for follow up after surgery, although, as I have indicated elsewhere, clinicians' fondness for follow up studies may have to yield to audits showing their lack of cost or benefit effectiveness. This would seem to be particularly true when women being treated with, for example, bromocriptine are asymptomatic as regards both their hormonal status and vision.

The greater sensitivity to both the internal structure of pituitary tumours and their relations with the anterior optic pathways make MRI the ideal method for investigating suspected pituitary apoplexy, and for deciding which patients actually need treatment, as the apoplectic episode may itself result in involution of tumour usually found to underlie it. ${ }^{105}$

Magnetic resonance imaging has opened doors to other endocrine disturbances to which CT was effectively blind. As an example, the normal posterior lobe of the pituitary gives high signal on T1 weighted MRI, for reasons not entirely clear. This is absent in most patients with central or nephrogenic diabetes insipidus, but not in those with primary polydipsia. ${ }^{106}$

\section{Conclusions}

It is difficult to draw overall conclusions from the foregoing, as I have tried to indicate in each case the sort of selection process that the neurologist or neurosurgeon should make when thinking of referring a patient for imaging. To best use his services to the patient's advantage, the clinician should: (a) refrain from any imaging for which there is no clear clinical indication and $(b)$ prefer anodyne to invasive, cheap to expensive, but (c) not perform a harmless, low cost examination if it will not obviate a more aggressive or expensive one.

In Britain the legal power to determine whether any imaging investigation is carried out is unequivocally vested in the radiologist. ${ }^{107}$ This gives force to the Royal College of Radiologists' uncontentious recommendation that "if you are in doubt as to whether an investigation is required or which investigation is best, it makes sense to ask the radiologists who, like other consultants, will know much more about their speciality than those whose primary interests are in other fields". ${ }^{20}$

1 Dumoulin CL, Hart HR. MR angiography. Radiology 1986;161:717-20.

2 Nitz WR, Bradley WG, Watanabe AS, Lee RR, Burgoyne B, O'Sullivan RM, et al. Flow dynamics of cerebrospinal fluid: assessment with phase-contrast velocity MR imaging performed with retrospective cardiac gating. imaging performed with retros

3 Gideon P, Sorensen PS, Thomsen C, Ståhlberg F, Gjerris $F$, Henriksen $O$. Assessment of CSF dynamics and venous flow in the superior sagittal sinus by MRI in idiopathic intracranial hypertension: a preliminary study. Neuroradiology 1994;36:350-4.

$4 \mathrm{McComb}$ JG. The usefulness of phase-contrast MR measurement of cerebrospinal fluid flow. AfNR Am $\mathcal{F}$ Neuroradiol 1993;14:1309-10.

5 Castillo M, Hudgins PA, Malko JA, Burrow BK, Hoffman JC Jr. Flow-sensitive MR imaging of ventriculoperiteonal shunts: in vitro findings, clinical applications, and piffalls. AfNR Am $\mathcal{F}$ Neuroradiol 1991;12:667-71.

6 Le Bihan D, Breton E, Lallemand D, Grenier P, Cabanis E Laval-Jeantet M. MR imaging of intravoxel incoherent 
motions: applications to diffusion and perfusion in neurological disorders Radiology 1988:161:401-7.

7 Henkelman RM. Diffusion-weighted MR imaging: a useful adjunct to clinical diagnosis or a scientific curiosity? AfNR Am $\mathcal{F}$ Neuroradiol 1990;12:932-4.

8 Dorothee O, Henning J, Ernst T. Human brain tumors: assessment with in vivo proton spectroscopy. Radiology 1993;186:745-52.

9 Vion-Dury J, Meyerhoff DJ, Cozzone PL, Weiner MW. What might be the impact on neurology of the analysis of brain metabolism by in vivo magnetic resonance spectroscopy? $¥$ Neurol 1994;241:354-71.

10 Rubin JM, Mirfakhree M, Duda EE, Dohrmann GJ, Brown F. Intraoperative ultrasound examination of the Brown F. Intraoperative ultrasou.
brain. Radiology 1980;137:831-2.

11 Counsell CE, Fraser H, Sandercock PAG. Archie Cochrane's challenge: can periodically updated reviews of all randomised controlled trials relevant to neurology of all randomised controlled trials relevant to neurology
and neurosurgery be produced? $₹$ Neurol Neurosurg and neurosurgery be produc

12 Daves ML. Radiologic overkill. $\mathcal{F} A M A$ 1967;200: 999-1000.

13 Kent DL, Larson EB. Magnetic resonance imaging of the brain and spine: is clinical efficacy established after the first decade? Ann Intern Med 1988;108:402-24.

14 Dixon AK, Southern JP, Teale A, Freer CEL, Hall LD, Williams A, et al. Magnetic resonance imaging of the head and spine: effective for the clinician or the patient? BMF 1991;302:79-82.

15 Friedman D, Rapaport R. Routine use of contrastenhanced MR scans in AIDS. AfNR Am $\mathcal{f}$ Neuroradiol 1993;14:1324.

16 Zimmerman RD. Routine use of contrast-enhanced MR scans in AIDS. AfNR Am $f$ Neuroradiol 1993;14: 1326-84.

17 McLean SAM. A patient's right to know. Information disclosure, the doctor and the law. Aldershot, Dartmouth, $1989 \cdot 14$.

18 Moseley IF. Long term effects of the introduction of noninvasive investigations in neuroradiology. Part 1: overal trends. Neuroradiology 1988;30:187-92.

19 Tatler GLV, Moseley IF. Use of invasive neuroradiological investigations in patients with normal computerised tomography. $B M \mp$ 1982;ii:1026-8.

20 Roberts GM, ed. Making the best use of a Department of Clinical Radiology. Guidelines for doctors 2 nd ed. London: Royal College of Radiologists, 1993.

21 Ansell G. Clinical considerations in the administration of contrast media to high-risk patients. In: Carr DH, ed. Contrast media. Edinburgh: Churchill Livingstone, 1988: 78-88.

22 Feuerman T, Wackym PA, Gade GF, Becker DP. Value of skull radiography, head computed tomographic scanning and admission for observation in cases of minor head injury. Neurosurgery 1988;22:449-53.

23 Hackney DB. Skull radiography in the evaluation of trauma: a survey of current practice. Radiology 1991; 181:711-4.

24 Colquhoun IR. CT cisternography in the investigation of cerebrospinal fluid rhinorrhoea. Clin Radiol 1993;47: 403-8.

25 Anon. Guidelines for the determination of death: report of the medical consultants on the diagnosis of death to the President's Commission for the study of ethical problems in medicine and biomedical and behavioral research. $¥ A M A$ 1981;246:2184-6.

26 Griner PF, Glaser RJ. Misuse of laboratory tests and diagnostic procedures. $N$ Engl $f$ Med 1982;207:1336-9.

27 Moseley IF. The plain radiograph in ophthalmology: a wasteful and potentially dangerous anachronism. $f R$ Soc wasteful and potentially

28 Moseley IF. The diagnostic value of "optic foramen views": experience from an eye hospital. $B r f$ Ophthalmo 1990;74:235-7.

29 Lewis TT, Kingsley DPE, Moseley IF. Do bilateral optic nerve meningiomas exist? Br f Neurosurg 1991;5:13-8.

30 Miller DH, Ormerod IEC, McDonald WI, ManManus DG, Kendall BE, Kingsley DPE, et al. The early risk of multiple sclerosis after optic neuritis. $\mathcal{F}$ Neurol Neurosur Psychiatry 1988;51:1569-71.

31 Guy J, Mancuso A, Beck R, Moster ML, Sedwick LA Quisling RG, et al. Radiation-induced optic neuropathy: a magnetic resonance imaging study. F Neurosurg 1991 74:426-32.

32 Steinsapir KD, Goldberg RA. Traumatic optic neuropathy. Surv Ophthalmol 1994;38:487-518.

33 Zimmerman C, Schatz NJ, Glaser JS. Magnetic resonance imaging of optic nerve meningiomas. Enhancement with imaging of optic nerve meningiomas. Enhancement
gadolinium-DPTA. Ophthalmology 1990;97:585-91.

34 Day AL. Aneurysms of the ophthalmic segment. A clinical and anatomical analysis. F Neurosurg 1990;72:677-91.

35 Jacobson DM, Karanja PN, Olson KA, Warner JJ. Computed tomography ventricular size has no value in diagnosis pseudotumor cerebri. Neurology 1990;40. 1454-5.

36 Bronstein AM, Rudge P, Gresty MA, du Boulay G, Morris J. Abnormalities of horizontal gaze and magnetic resonance findings. II. Gaze palsy and internuclear ophthalmoplegia. F Neurol Neurosurg Psychiatry 1990;53 200-7.

37 Schwaber MK, Larson TC, Zealear DL, Creasy J Gadolinium-enhanced magnetic resonance imaging in Bell's palsy. Laryngoscope 1990;100:1264-9.

38 Anderson RE, Laskoff JM. Ramsay Hunt syndrome mimicking intracanalicular acoustic neuroma on contrast-enhanced MR. AFNR Am F Neuroradiol 1990; 11:409.

39 Anon. Imaging patients with suspected acoustic neuroma. Lancet 1988;ii: 1294

40 Welling DB, Glasscock ME, Woods CI, Jackson CG. Acoustic neuroma: a cost-effective approach. Otolaryngol Head Neck Surg 1990;103:364-70.

41 Consensus Development Panel. National Institutes of Health Consensus Development Conference statement on acoustic neuroma, 11-13 December 1991. Arch Neurol 1991;51:201-7.

42 Renowden SA, Anslow P. The effective use of magnetic resonance imaging in the diagnosis of acoustic neuromas. Clin Radiol 1993;48:25-8.

43 Bernardi B, Zimmerman RA, Savino PJ, Adler C. Magnetic resonance tomographic angiography in the
investigation of hemifacial spasm. Neuroradiology 1993; investigation

44 Bradley WG. MR of the brain stem: a practical approach. Radiology 1991;179:319-32.

45 Savy LE, Stevens JM, Taylor DJ, Kendall BE. Apparent cerebellar ectopia: a reappraisal using volumetric MRI Neuroradiology 1994;36:360-3.

46 Asbury AK, Herndon RM, McFarland HF, McDonald WI, McIlroy WJ, Paty DW, et al. National Multiple Sclerosis Society working group on neuroimaging for the Medical Advisory Board. Neuroradiology 1987;29:119.

47 Miller DH, Ormerod IEC, Rudge P, Kendall BE, Moseley IF, McDonald WI. The early risk of multiple sclerosis following isolated syndromes of the brainstem and spinal
cord. Ann Neurol 1989;26:635-9.

48 Rudge P, Ali A, Cruickshank JC. Multiple sclerosis, tropical spastic paraparesis, and HTLV-1 infection in AfroCaribbean patients in the United Kingdom. $\mathcal{f}$ Neurol Neurosurg Psychiatry 1991;54:689-94.

49 Miller DH, Ormerod IEC, Gibson A, du Boulay EPGH, Rudge P, McDonald WI. MR brain scanning in patients Rudge $P$, McDonald WI. MR brain scanning in patients
with vasculitis: differentiation from multiple sclerosis. with vasculitis: differentiation

50 Banna M, El-Ramahi K. Neurologic involvement in Behcet disease: imaging findings in 16 patients. AfNR Am $\mathcal{f}$ Neuroradiol 1991;12:791-6.

51 Fazekas F, Offenbacher H, Fuchs S, Schmidt R, Niederkorn K, Horner S, et al. Criteria for an increased specificity of MRI interpretation in elderly subjects with

52 Viñuela FV, Fox AJ, Debrun GM, Feasby TE, Ebers GC. New perspectives in computed tomography of multiple sclerosis. Am ₹ Radiol 1982;139:123-7.

53 Kesselring J, Miller DH, Robb SA, Kendall BE, Moseley $\mathrm{IF}$, Kingsley $\mathrm{D}$, et al. Acute disseminated encephalomyelitis. MRI findings and the distinction from multiple myelitis. MRI findings and the distinct

54 Lunsford LD, Martinez AJ, Latchaw RE, Pazin GJ. Rapid and accurate diagnosis of herpes simplex encephalitis with computed tomographic stereotaxic biopsy. Surg weurol 1984;21:249-57.

55 Fernandez RE, Rothberg M, Ferencz G, Wujack D. Lyme disease of the CNS: MR imaging findings in 14 cases. AfNR Am $\mathcal{F}$ Neuroradiol 1990;11:479-81.

56 Tien RD, Chu PK, Hesselink JR, Duberg A, Clayton W intracranial cryptococcosis in immunocompromised Neuroradiol 1991;12:283-9.

57 Mercader JM, Perich J, Berenguer J, Pujol T, Cardenal C. Intracranial tuberculoma in AIDS: neuroradiological findings. Neuroradiology 1991;33:569-70.

58 Dina TS. Primary central nervous system lymphoma versus toxoplasmosis in AIDS. Radiology 1991;179: 823-8.

59 Moseley IF. Radiological investigation of cerebral masses. Current Imaging 1991;3:31-6.

60 Hillemacher A. Die Wert amnesticher and klinische Daten sowie apparativer Intersuchundsbefunde bei der
Diagnose von Hirntumoren. Fortschr Neurol Psychiatr Diagnose von Hirn

61 Baker HL, Houser OW, Campbell JK. National Cancer Institute study: evaluation of computed tomography in the diagnosis of intracranial neoplasms. I. Overal results. Radiology 1980;136:91-6.

62 Haughton HV, Rimm AA, Sobocinski KA, Papke RA, Daniels DL, Williams AL, et al. Blinded clinical comparison of MR imaging and $\mathrm{CT}$ in neuroradiology. Radiology 1986;160:751-5.

63 Kazner E, Wende S, Grumme T, Lanksch W, Stochdorph O. Computed tomography in intracranial tumours. Berlin Springer-Verlag, 1982.

64 Bolender NF, Cromwell LD, Graves V, Margolis MT, Kerber CW, Wendling $\mathrm{L}$. Interval appearance of glioblastoma not evident in previous CT examination. glioblastoma not evident in previous C

65 Enzmann DR, Krikorian J, Yorke C, Hayward R. Computed tomography in leptomeningeal spread of Computed tomography in leptomeningeal spr

66 Sze G, Milano E, Johnson C, Heier L. Detection of brain metastases: comparison of contrast-enhanced MR with
unenhanced MR and enhanced CT. AfNR Am $\mathcal{f}$ Neuroradiol 1990;11:785-91.

67 Ruggiero G, Picard L. The true value of computed tomography, angiography and pneumoencephalography for the precise diagnosis of the brain tumours. In: Wackenheim $\mathrm{A}$, du Boulay GH (eds) Choices and characteristics in computerized tomography. Amsterdam: Kugler, 1980:13-21.

68 De Angelis LM. Primary nervous system lymphoma: a new clinical challenge. Neurology 1991;41:619-21. 
69 Davies AG, Richardson RB, Bourne SP, Kemshead JT, Coakham HB. Immunolocalisation of human brain tumours. In: Bleehen NM, ed. Tumours of the brain. Berlin: Springer-Verlag, 1986, 83-99.

70 Asari S, Makabe T, Katayama S, Itoh T, Tsuchida S, Ohmoto $T$. Assessment of the pathological grade of astrocytic gliomas using an MRI score. Neuroradiology 1994;36:308-10.

71 Jelinek J, Smirniotopoulos JG, Parisi JE, Kanzer M. Lateral ventricular neoplasms of the brain: differential diagnosis based on clinical, CT and MR findings. AFNR Am $\mathcal{F}$ Neuroradiol 1990;11:567-74.

72 Peeling J, Sutherland G, Marat K, Tomchuk E, Bock E. $1 \mathrm{H}$ and $13 \mathrm{C}$ nuclear magnetic resonance studies of plasma from patients with primary intracranial neoplasma from patients with primary

73 Carpeggiani $P$, Crisi G, Trevisan C. MRI of intracranial meningiomas: correlations with histology and physical meningiomas: correlations with histology

74 Brismar J, Cronqvist S. Therapeutic embolisation in the external carotid artery region. Acta Radiologies 1978; 19:715-31.

75 Halbach VV, Hieshima GB, Higashida RT, David CF. Endovascular therapy of head and neck tumors. In Viñuela F, Halbach VV, Dion JE, eds. Interventional neuroradiology. Endovascular therapy of the central nervous system. New York: Raven Press, 1992:17-28.

76 Bergstrom M, Collins P, Ehrin E, Ericson K, Ericsson L, Greitz T, et al. Discrepancies in brain tumour extent as shown by computed tomography and positron emission tomography using [ $\left.{ }^{68 \mathrm{Ga}}\right] \mathrm{EDTA},\left[{ }^{11} \mathrm{C}\right]$ glucose, and ["C]methionine. F Comput Assist Tomogr 1983;7: [1062-6.

77 Piani C, Pasquini U, De Nicola $M$, Bevilacqua $F$ Menichelli F, Salvolini U, et al. Intraarterial ACNU chemotherapy in malignant cerebral gliomas: preliminchemotherapy in malignant cerebral gliomas:
ary results. Neuroradiology 1991;33:319-21.

78 Schwartz RB, Carvalho PA, Alexander E III, Loeffler JS, Folkerth R, Holman BL. Radiation necrosis vs highgrade recurrent glioma: differentiation by using dual-
isotope SPECT with ${ }^{201} \mathrm{~T} 1$ and ${ }^{99 \mathrm{~m}} \mathrm{Tc}-\mathrm{HMPAO}$. AfNR Am $\mathcal{f}$ Neuroradiol 1991;12:1 187-92.

79 Larson EB, Omenn GS, Lewis H. Diagnostic evaluation of headache. Impact of computerised tomography and cost-effectiveness. FAMA 1980;243:359-62.

80 Heller CA, Stanley P, Lewis-Jones B, Heller RF. Value of $x$ ray examinations of the cervical spine. $B M F$ 1983;287: 1276-8.

81 Daras M, Tuchman AJ, Strobos RJ. Computed tomography in adult onset epilepsy. Can $\mathcal{F}$ Neurol Sci 1987; 14:286-9.

82 Morrell $F$. In vivo imaging of human anatomy in temporal lobe epilepsy. AFNR Am 7 Neuroradiol 1991;12: 948-9.

83 Orrison WW, Davis LE, Sullivan GW, Mettler FA Jr, Flynn ER. Anatomic localization of cerebral cortical function by magnetoencephalography combined with MR imaging and CT. AFNR Am $\mathcal{F}$ Neuroradiol 1990; 11:713-6.

84 Borbely K, Balogh A, Halasz P, Vajda J, Czirjak S, Nyary I. Reliability of ictal and peri-ictal SPECT investigations in localizing epileptogenic foci in patients undergoin epilepsy surgery. Neuroradiology 1994;36(suppl):S76.

85 Drayer BP, Olanow W, Burger P, Johnson GA, Herfkens $\mathrm{R}$, Riederer S. Parkinsonism plus syndrome: diagnosis using high field MR imaging of brain iron. Radiology 1986;159:493-8.

86 Savoiardo M, Halliday WC, Nardocci N, Strada L D'Incerti L, Angelini L, et al. Hallervorden-Spatz disease: $M R$ and pathological findings. AfNR Am $f$ Neuroradiol 1993;14:155-62.

87 Nazer H, Brismar J, Al-Kawi MZ, Gunasekaran TS, Jorulf $\mathrm{KH}$. Magnetic resonance imaging of the brain in KH. Magnetic resonance imaging of the
Wilson's disease. Neuroradiology 1993;35:130-3.

88 Savoiardo M, Strada L, Girotti F, Zimmerman RA, Grisoli
M, Testa $\mathrm{D}$, et al. Olivopontocerebellar atrophy: MR diagnosis and relationship to multisystem atrophy. Radiology 1990;174.693-6.

89 Bradshaw JR, Thomson JLG, Campbell MJ. Computed tomography in the investigation of dementia. $B M^{\mathcal{J}}$ 1983;286:277-80.

90 Ettun TM, Staehelin HB, Kischka U, Ulrich J, ScolloLavizzari G, Wiggli U, et al. Computed tomography, electroencephalography and clinical features in the differential diagnosis of senile dementia. Arch Neurol 1989;46:1217-20.

91 Early B, Rodrigo Escalona P, Boyko OB, Doraiswamy PM, Axelson DA, Patterson L, et al. Interuncal distance in healthy volunteers and in patients with Alzheimer disease. AfNR Am $\mathcal{f}$ Neuroradiol 1993; 14:907-10.

92 Jobst KA, Smith AD, Szatmari M, Molyneux A, Esiri $M E$, King $E$, et al. Detection in life of confirmed ME, King E, et al. Detection in life of confrmed Alzheimer's disease using a simple measurement of temporal lobe atrophy

93 Erkinjuntti T, Gao F, Lee DH, Eliasziw M, Merskey $H$, Hachinski VC. Lack of difference in brain hyperintensities between patients with early Alzheimer's disease and control subjects. Arch Neurol 1994;51:260-8.

94 Steiner I, Gomori JM, Melamed E. Features of brain atrophy in Parkinson's disease. Neuroradiology 1985;27:

95 Huber SJ, Shuttleworth EC, Christy JA, Chakeres DW, Curtin A, Paulson GW. Magnetic resonance imaging in dementia of Parkinson's disease. I Neurol Neurosur Psychiatry 1989;52:1221-7.

96 Callanan MM, Logsdail SJ, Ron MA, Warrington ER. Cognitive impairment in patients with clinically isolated lesions of the type seen in multiple sclerosis. Brain lesions of the type

97 Wardlaw JM, Sellar RJ. Early caudate nucleus atrophy in Huntington's disease: does it correlate with presenting Huntington's disease: does it correlate with
symptoms? Neuroradiology 1991;33:238-40.

98 Wakhloo AK, Jüngling $F$, Krauss JK, Schumacher $M$, Hennig J. Measurements of pulsatile brain motion with MR-interferography in patients with NPH. Neuroradiology 1992;34(suppl):S47.

99 Bradley WG Jr, Whittemore AR, Watanabe AS, Davis SJ Teresi LM, Homyak M. Association of deep white matter infarction with chronic communicating hydrocephalus: implications regarding the possible origin of normal-pressure hydrocephalus. AFNR $A m^{\mathcal{F}}$ Neuroradiol 1991;12:31-9.

100 George AE. Chronic communicating hydrocephalus and periventricular white matter disease: a debate with regard to cause and effect. AłNR Am $₹$ Neuroradiol 1991;12:42-4.

101 Moseley I. Neuropsychiatric disease. Curr Opin Neurol Neurosurg 1990;3:884-9.

102 Andreasen NC. Neuroradiology and neuropsychiatry: a new alliance. AfNR Am $\mathcal{F}$ Neuroradiol 1992;13:841-3.

103 John alliance. AfNR Am F Neuroradiol 1992;13:841-3. Llewellyn DEH, et al. The evaluation of patients with a suspected pituitary microadenoma: computed tomography compared to magnetic resonance imaging. Clin Endocrinol 1992;36:335-8.

104 Marcovitz S, Wee R, Chan J, Hardy J. The diagnostic accuracy of preoperative $\mathrm{CT}$ scanning in the evaluation of pituitary ACTH-secreting adenomas. AfNR $A m$ Neuroradiol 1987;8:641-4.

105 L'Huillier F, Combes C, Martin N, Leclerc X, Pruco JP, Gaston A. MRI in the diagnosis of so-called pituitary apoplexy. I Neuroradiol 1989;16:221-37.

106 Moses AM, Clayton B, Hochhauser L. Use of T1 weighted $M R$ imaging to differentiate between primary weighted MR imaging to differentiate between primary polydipsia and central diabetes

107 Moseley IF. Diagnostic imaging in neurological disease. Edinburgh: Churchill Livingstone, 1986:1-2. 